\title{
Doenças pós-colheita em laranja 'Pêra' produzida em sistema orgânico e convencional e resistência de Penicillium digitatum a fungicidas
}

\author{
Ivan Herman Fischer ${ }^{1}$, Maria Cecília de Arruda Palharini ${ }^{1}$, Marcel Bellato Spósito² \& Lilian Amorim ${ }^{3}$
}

${ }^{1}$ Laboratório de Fitossanidade, APTA Centro Oeste, 17030-000, Bauru, SP, Brasil; ${ }^{2}$ Departamento de Produção Vegetal, ESALQ, Universidade de São Paulo, 13418-900, Piracicaba, SP, Brasil; ${ }^{3}$ Departamento de Fitopatologia e Nematologia, ESALQ, Universidade de São Paulo, 13418-900, Piracicaba, SP, Brasil

Autor para correspondência: Ivan Herman Fischer (ihfische@apta.sp.gov.br)

Data de chegada: 21/10/2011. Aceito para publicação em: 21/01/2013.

\section{RESUMO}

Fischer, I.H.; Palharini, M.C.A.; Spósito, M.B.; Amorim, L.. Doenças pós-colheita em laranja 'Pêra' produzida em sistema orgânico e convencional e resistência de Penicillium digitatum a fungicidas. Summa Phytopathologica, v.39, n.1, p.28-34, 2013.

As doenças pós-colheita podem ser responsáveis por perdas significativas ao citricultor. Este trabalho objetivou: a) caracterizar as doenças pós-colheita em laranjas 'Pêra' provenientes de cultivo orgânico e convencional, com ou sem tratamento pós-colheita com imazalil, comercializadas na CEAGESP; e b) detectar a presença de isolados de Penicillium digitatum resistentes a fungicidas em frutos com bolor verde. Frutos permaneceram em câmara úmida por 24 horas, e por mais 13 dias a $25{ }^{\circ} \mathrm{C}$ e $85 \%$ de UR. A incidência de doenças foi avaliada visualmente a cada 2-3 dias. A presença de $P$. digitatum resistente a fungicidas foi avaliada em função do crescimento micelial em meio batata-dextrose-ágar acrescido dos fungicidas tiabendazol $\left(10 \mathrm{mg} . \mathrm{L}^{-1}\right)$ e imazalil $\left(1 \mathrm{mg} . \mathrm{L}^{-1}\right)$. Frutos tratados com imazalil apresentaram a menor incidência $(5,4 \%)$ de doenças póscolheita, enquanto que frutos provenientes de pomares orgânicos apresentaram a maior incidência $(25,2 \%)$. As principais doenças póscolheita foram o bolor verde em frutos produzidos em sistema convencional e as podridões pedunculares de lasiodiplodia e de phomopsis em frutos orgânicos. A frequência relativa de isolados de $P$. digitatum resistentes ao fungicida tiabendazol foi menor em frutos orgânicos, enquanto a frequência de isolados de $P$. digitatum resistentes ao imazalil e à mistura tiabendazol+imazalil foi maior em frutos que receberam tratamento pós-colheita com imazalil.

Palavras-chave adicionais: Citrus sinensis, podridões, bolor verde, resistência a fungicidas.

\section{ABSTRACT}

Fischer, I.H.; Palharini, M.C.A.; Spósito, M.B.; Amorim, L.. Postharvest diseases in 'Pêra' orange cultivated in organic and conventional systems and resistance of Penicillium digitatum to fungicides. Summa Phytopathologica, v.39, n.1, p.28-34, 2013.

Postharvest diseases may be responsible for significant losses to citrus growers. This study aimed to: a) characterize the postharvest diseases in 'Pêra' oranges from organic and conventional crops, with or without postharvest treatment with imazalil, marketed in CEAGESP; and b) detect the presence of Penicillium digitatum isolates resistant to fungicides in fruits with green mold. Fruits were kept in a humid chamber for 24 hours and for additional 13 days at $25{ }^{\circ} \mathrm{C}$ and $85 \%$ RH. The disease incidence was visually evaluated at every $2-3$ days. The presence of $P$. digitatum resistant to the fungicides was assessed based on mycelial growth on potato-dextrose-agar medium added of the fungicides thiabendazole $\left(10 \mathrm{mg} . \mathrm{L}^{-1}\right)$ and imazalil (1 mg. $\left.\mathrm{L}^{-1}\right)$. Fruits treated with imazalil had lower incidence $(5.4 \%)$ of postharvest diseases, while fruits from organic crop had the highest incidence $(25.2 \%)$. The main postharvest diseases were green mold in fruits produced in the conventional system and Lasiodiplodia and Phomopsis stem-end rot in organic fruits. The relative frequency of $P$. digitatum isolates resistant to the fungicide thiabendazole was lower in organic fruits, while the frequency of $P$. digitatum isolates resistant to imazalil and the mixture thiabendazole+imazalil was higher in fruits that received postharvest treatment with imazalil.

Additional keywords: Citrus sinensis, rots, green mold, fungicide resistance

Os danos em pós-colheita de citros são causados principalmente por patógenos fúngicos, normalmente decorrentes de práticas inadequadas de condução da cultura no campo e durante a colheita, transporte e/ou embalagem. Em levantamento das doenças pós-colheita em laranjas 'Pêra' comercializadas na Central de Abastecimento de Recife detectou-se $22 \%$ de incidência após sete dias de armazenamento (7). Em frutos cítricos procedentes de packinghouse paulista visando o mercado interno, a incidência de doenças pós-colheita foi superior a $14 \%$, após duas semanas de armazenamento a $25{ }^{\circ} \mathrm{C}(9)$, entretanto, em packinghouse com frutos destinados à exportação, a incidência ficou abaixo de $3 \%$, evidenciando diferenças na qualidade fitossanitária do fruto e as boas práticas agrícolas adotadas, que incluem tratamento com $\mathrm{NaCl}$ e fungicidas em pós-colheita (11).

O bolor verde (Penicillium digitatum) é considerado a principal doença pós-colheita dos citros, principalmente em climas quentes. A infecção pelo patógeno ocorre por meio de ferimentos onde os nutrientes estão disponíveis e estimulam a germinação dos esporos depositados na superfície do fruto (18). Em São Paulo, o bolor verde 
foi considerado a principal doença pós-colheita em frutos de laranjas 'Pêra', 'Lima' e 'Natal' e em tangor 'Murcott', comercializados no mercado interno (9). Outras doenças, como as podridões pedunculares de lasiodiplodia (Lasiodiplodia theobromae) e phomopsis (Phomopsis citri), a antracnose (Colletotrichum gloeosporioides), o bolor azul ( $P$. italicum) e a podridão negra (Alternaria alternata) podem ser importantes sob determinadas condições (18).

O controle do bolor verde é baseado principalmente no uso dos fungicidas em pós-colheita, especialmente tiabendazol e imazalil, aplicados isoladamente ou em mistura nos packinghouses $(3,11)$. Estudos vêm constatando que o fracasso de muitos tratamentos com fungicidas em pós-colheita decorre do fenômeno de resistência por parte dos patógenos. Estes fungicidas são empregados de modo altamente condutivo à seleção e proliferação de biótipos resistentes de Penicillium. A superfície do fruto é completamente coberta pelos fungicidas e o resíduo é persistente com a vida do fruto. Muitos packinghouses processam frutos ininterruptamente durante o ano, resultando em contínua pressão de seleção na população do patógeno (14). Em levantamento da frequência de isolados de $P$. digitatum resistentes a tiabendazol em frutos que chegavam ao porto de Rotterdam, na Holanda, constatou-se a presença de isolados resistentes em frutos com sintomas de bolor verde provenientes de 18 países, inclusive do Brasil com 33\% de isolados resistentes (19). A proporção de isolados de $P$. digitatum resistentes simultaneamente aos fungicidas tiabendazol, ortofenilfenato de sódio e imazalil aumentou de $43 \%$ em 1988 para 77\% em 1990, em packinghouses californianos (15). Amostragens feitas no ambiente de packinghouses paulistas constataram que $39 \%$ dos isolados de $P$. digitatum apresentavam resistência ao fungicida tiabendazol e $1 \%$ ao imazalil (12).

À medida que se tem questionado a aplicação de fungicidas sintéticos na agricultura, pelos riscos à saúde e ao meio ambiente, vem crescendo a demanda por alimentos mais saudáveis, produzidos em sistemas de cultivo agroecológico, como o orgânico, valorizando a diversidade biológica e sem o uso de fertilizantes químicos e agrotóxicos. O sistema de cultivo orgânico de citros vem sendo adotado por produtores de vários estados brasileiros, sendo São Paulo o maior produtor (6). Apenas na região de Itápolis, SP, há mais de 30 produtores que processam e exportam suco de laranja orgânico para a União Européia e Estados Unidos. Entretanto, segundo Sartori et al. (23), o principal entrave para a produção de citros orgânico é a falta de conhecimento científico para o cultivo orgânico. Com a intensificação da vida microbiológica do solo e ambiental e o aumento da biodiversidade, relatada nos sistemas de cultivo orgânicos $(13,20)$, espera-se estabelecer o controle biológico de pragas e doenças. No entanto, desconhece-se o impacto do manejo incentivado na agricultura orgânica sobre as doenças pós-colheita dos citros.

O presente trabalho teve por objetivos: a) caracterizar as doenças pós-colheita em frutos de laranja doce 'Pêra' provenientes de cultivo orgânico e convencional, com ou sem tratamento com imazalil em póscolheita, comercializados na Companhia de Entrepostos e Armazéns Gerais de São Paulo (CEAGESP); e b) detectar a presença de isolados de Penicillium spp. resistentes aos fungicidas tiabendazol e imazalil nos frutos com bolor verde.

\section{MATERIAL E MÉTODOS}

\section{Doenças pós-colheita}

Cem frutos de laranja 'Pêra' (Citrus sinensis Osbeck) foram coletados mensalmente, de julho a dezembro de 2008 e 2009, em seis atacadistas da CEAGESP, onde dois comercializam frutos produzidos em sistema convencional sem tratamento com fungicida em póscolheita, dois comercializam frutos tratados com o fungicida imazalil no packinghouse e dois comercializam frutos produzidos em sistema orgânico. Os municípios de procedência dos frutos amostrados durante todo o experimento estão mencionados na Tabela 1. Os frutos produzidos em sistema convencional foram comercializados em caixas de madeira tipo $\mathrm{M}$, enquanto os frutos orgânicos em caixas de papelão ou engradados plásticos.

Os frutos foram individualizados em bandejas plásticas e submetidos à câmara úmida por 24 horas, visando favorecer a ocorrência de doenças, permanecendo por mais 13 dias a $25^{\circ} \mathrm{C}$ e $85 \%$ de umidade relativa, em câmara de crescimento na APTA-Bauru. A incidência de doenças foi avaliada visualmente a cada dois-três dias e os fungos isolados de frutos doentes tiveram a patogenicidade confirmada por meio de inoculação em frutos sadios. Os dados de incidência de doenças nos sistemas de cultivo e entre os sistemas de cultivo foram comparados por teste não paramétrico de comparação de múltiplas proporções ao nível de $5 \%$ de probabilidade (29).

\section{Determinação de isolados de Penicillium spp. resistentes a fungicidas}

Frutos de laranja 'Pêra' com sintomas de bolor verde tiveram os conídios de $P$. digitatum repicados, com auxílio de uma agulha histológica, da superfície de frutos doentes para placas de Petri contendo quatro meios de cultura distintos, acrescidos de pentabiótico $\left(0,4\right.$ g. $\left.\mathrm{L}^{-1}\right)$ : batata-dextrose-ágar (BDA), BDA com $10 \mathrm{mg} . \mathrm{L}^{-1} \mathrm{de}$ ingrediente ativo (i.a.) de tiabendazol, BDA com $1 \mathrm{mg} . \mathrm{L}^{-1}$ de i.a. de imazalil e BDA com os dois fungicidas $\left(10 \mathrm{mg} . \mathrm{L}^{-1}\right.$ de i.a. de tiabendazol $+1 \mathrm{mg} . \mathrm{L}^{-1}$ de i.a. de imazalil). Estas doses de fungicidas correspondem às chamadas doses de resistência, obtidas experimentalmente, e nas quais se considera que unicamente os isolados resistentes são capazes de crescer $(4,15,30)$. O tiabendazol (Tecto SC) e o imazalil (Magnate $500 \mathrm{CE}$ ), assim como o pentabiótico (Fort Dodge ${ }^{\circledR}$ ), foram adicionados ao meio de cultura BDA fundente, em câmara de ar de fluxo laminar. Para cada fruto doente e meio de cultura utilizados foram empregadas três placas de Petri (repetições).

As incidências de isolados de P. digitatum resistentes aos fungicidas foram comparadas por teste não paramétrico de múltiplas proporções ao nível de $5 \%$ de probabilidade (29). Os dados de crescimento micelial destes isolados em meio de cultura acrescido de fungicidas foram submetidos à análise de variância (Tukey, $P<0,05$ ), visando estudar possíveis diferenças entre os sistemas de cultivo convencional e orgânico.

\section{RESULTADOS E DISCUSSÃO}

\section{Doenças pós-colheita}

A incidência total de doenças pós-colheita em laranja 'Pêra' comercializada na CEAGESP foi menor nos frutos que receberam tratamento pós-colheita com imazalil e maior nos frutos orgânicos em relação aos do sistema convencional que não receberam o tratamento fungicida, ao final dos 14 dias de armazenamento, e as doenças mais frequentes foram, na média, o bolor verde $(5,88 \%)$ e a podridão peduncular de lasiodiplodia (4,26\%), seguidas pela podridão peduncular de phomopsis (3,37\%) (Tabela 2). A incidência do bolor verde foi maior em frutos do sistema convencional que não receberam tratamento pós-colheita com imazalil, a partir da primeira semana de armazenamento (Figura 1), e as incidências das podridões pedunculares 
Tabela 1. Procedências (minicípio, estado) dos frutos cítricos amostrados em atacadistas na CEAGESP, em 2008 e 2009.

\begin{tabular}{|c|c|c|c|c|}
\hline \multirow{2}{*}{$\begin{array}{l}\text { Meses de amostragem } \\
\text { (sistema de cultivo) }\end{array}$} & \multicolumn{2}{|c|}{2008} & \multicolumn{2}{|c|}{2009} \\
\hline & \multicolumn{4}{|c|}{ Atacadistas } \\
\hline Convencional+imazalil & 1 & 2 & 1 & 2 \\
\hline Julho & Colômbia-SP & Casa Branca-SP & Votuporanga-SP & Urânia-SP \\
\hline Agosto & Lucianópolis-SP & Casa Branca-SP & Pratânia-SP & Botucatu-SP \\
\hline Setembro & Barretos-SP & Eng. Coelho-SP & Bebedouro-SP & Urânia-SP \\
\hline Outubro & Bariri-SP & Eng. Coelho-SP & Altinópolis-SP & Frutal-SP \\
\hline Novembro & Pratânia-SP & Itumbiara-GO & Bebedouro-SP & Jales-SP \\
\hline Dezembro & Pratânia-SP & Turmalina-SP & Bebedouro-SP & Jales-SP \\
\hline \multicolumn{5}{|l|}{ Convencional } \\
\hline Julho & Limeira-SP & Bariri-SP & Casa Branca-SP & Bariri-SP \\
\hline Agosto & Pirangi-SP & Mogi Guaçu-SP & Ubirajara-SP & Pirangi-SP \\
\hline Setembro & Jales-SP & Bariri-SP & Avaré-SP & Bariri-SP \\
\hline Outubro & Araras-SP & Tambaú-SP & Cosmorama-SP & Bariri-SP \\
\hline Novembro & Araraquara-SP & Bariri-SP & Pirangi-SP & Pirangi-SP \\
\hline Dezembro & Pirangi-SP & Bariri-SP & Pirassununga-SP & Bariri-SP \\
\hline \multicolumn{5}{|l|}{ Orgânica } \\
\hline Julho & Monte Alto-SP & - & Gonçalves-MG & - \\
\hline Agosto & Mogi Guaçu-SP & - & Rio Real-BA & Guararema-SP \\
\hline Setembro & Mogi Guaçu-SP & - & Rio Real-BA & - \\
\hline Outubro & Mogi Guaçu-SP & - & Rio Real-BA & - \\
\hline Novembro & Mogi Guaçu-SP & - & Jales-SP & - \\
\hline Dezembro & Mogi Guaçu-SP & - & Bebedouro-SP & - \\
\hline
\end{tabular}

foram maiores em frutos orgânicos, já a partir do quarto dia de armazenamento (Figura 1). Frutos que receberam tratamento com imazalil em pós-colheita apresentaram menor incidência de bolor verde e de podridão peduncular de lasiodiplodia em relação aos frutos não tratados ao final do armazenamento. A incidência de antracnose foi superior em frutos orgânicos $(2,17 \%)$. Outras doenças foram observadas em menores incidências $(<1,0 \%)$, como a podridão de fusarium, a podridão azeda, o bolor azul e a podridão negra.

Diferenças na incidência total de doenças pós-colheita em laranjas 'Pêra' foram observadas entre os meses amostrados (Figura 2), relacionadas, provavelmente, às diferentes condições ambientais e procedências dos frutos em cada mês de amostragem. A frequência e a intensidade das doenças são fortemente influenciadas pela variação ambiental imposta durante todas as etapas da pré e pós-colheita (1). Diferenças na incidência das doenças entre os períodos de coleta de frutos na CEAGESP, também foram verificadas em amostragens em packinghouses e centrais atacadistas por Dantas et al. (7) e Fischer et al. $(9,10)$. Em adição, observou-se em 2009 um aumento expressivo na incidência de doenças, em relação a 2008 , de $72,5 \%$ em laranja 'Pêra' convencional+imazalil a 272,0\% em laranja 'Pêra' orgânica (dados

Tabela 2. Incidência (\%) ${ }^{1}$ de doenças pós-colheita em laranjas 'Pêra' comercializadas na CEAGESP, produzidas em sistema orgânico e convencional, aos 14 dias de armazenamento a $25^{\circ} \mathrm{C}$.

\begin{tabular}{|c|c|c|c|c|}
\hline Doenças & \multicolumn{4}{|c|}{ Sistema de cultivo } \\
\hline Bolor verde & $2,85 \mathrm{cA}^{3}$ & 9,96 aA & $4,83 \mathrm{bB}$ & $5,88 \mathrm{~A}$ \\
\hline Podridão de lasiodiplodia & $0,98 \mathrm{cB}$ & $2,88 \mathrm{bB}$ & $8,92 \mathrm{aA}$ & $4,26 \mathrm{AB}$ \\
\hline Podridão de phomopsis & $0,69 \mathrm{bBC}$ & $1,29 \mathrm{bBC}$ & 8,13 aA & $3,37 \mathrm{~B}$ \\
\hline Antracnose & $0,29 \mathrm{bBCD}$ & $0,71 \mathrm{bCD}$ & $2,17 \mathrm{aC}$ & $1,06 \mathrm{C}$ \\
\hline Podridão azeda & $0,13 \mathrm{aCD}$ & $0,29 \mathrm{aDE}$ & $0,17 \mathrm{aDE}$ & $0,19 \mathrm{CD}$ \\
\hline Bolor azul & $0,15 \mathrm{aBCD}$ & $0,04 \mathrm{aE}$ & $0,13 \mathrm{aDE}$ & $0,10 \mathrm{D}$ \\
\hline Podridão negra & $0,04 \mathrm{aD}$ & $0,04 \mathrm{aE}$ & $0,17 \mathrm{aE}$ & $0,08 \mathrm{D}$ \\
\hline Total & $5,44 \mathrm{c}$ & $15,38 \mathrm{~b}$ & $25,25 \mathrm{a}$ & 15,35 \\
\hline
\end{tabular}

${ }^{1}$ Média de doze amostragens mensais (jullho a dezembro de 2008 e 2009) de 200 frutos do sistema de cultivo convencional e 100 frutos orgânicos. ${ }^{2}$ Tratamento em pós-colheita com imazalil (1000 mg L L $).{ }^{3}$ Valores seguidos pela mesma letra, minúscula na linha e maiúscula na coluna, não diferem entre si, em nível de $5 \%$ pelo teste não paramétrico de comparação de múltiplas proporções (Zar, 1999). 

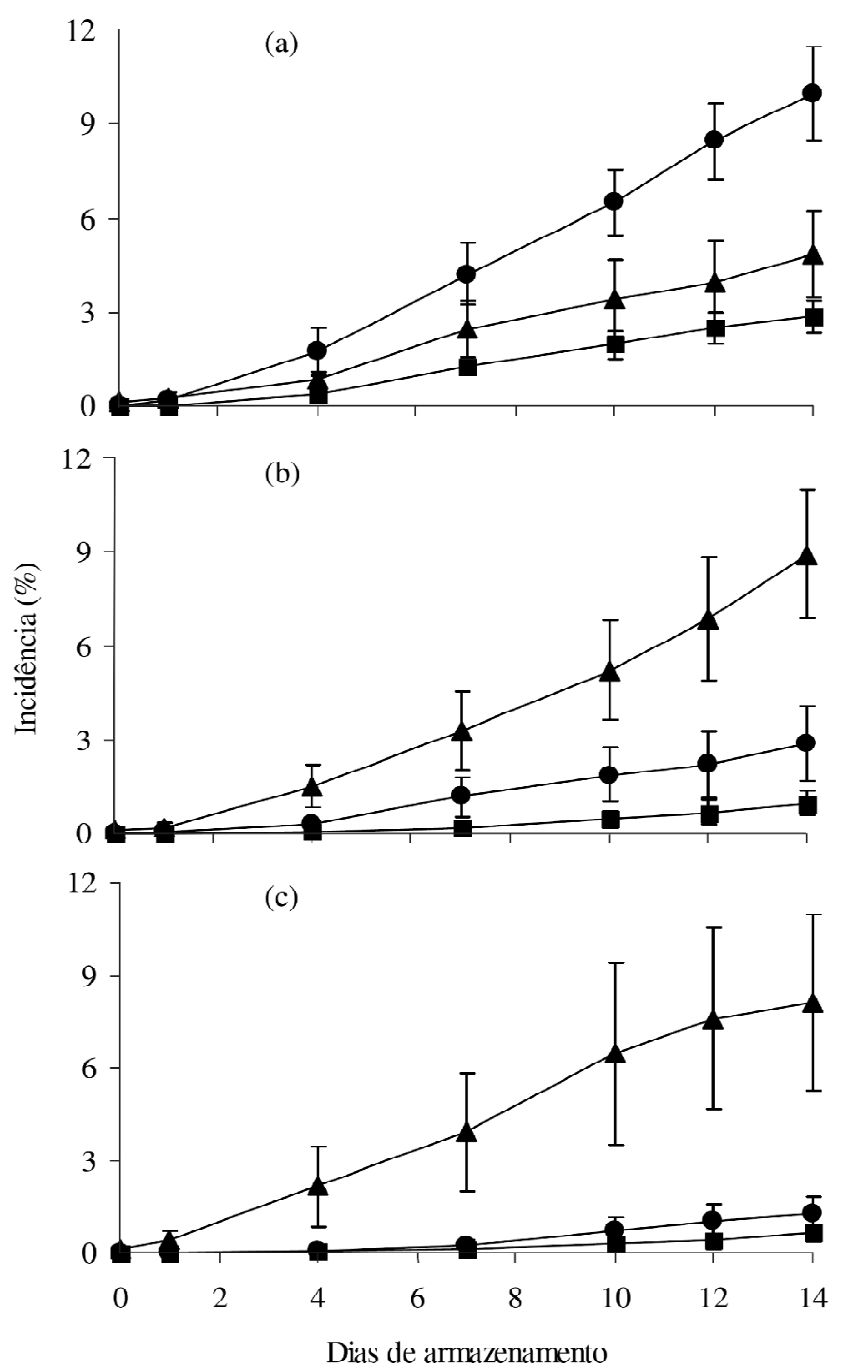

$\rightarrow$ - Convencional + Imazalil $\rightarrow$ Convencional $\rightarrow$ Orgânico

Figura 1. Progresso da incidência (\%) do bolor verde (a) e das podridões pedunculares de lasiodiplodia (b) e phomopsis (c) em laranjas 'Pêra' comercializadas na CEAGESP (média de 2008 e 2009), produzidas em sistema orgânico, convencional e convencional com tratamento póscolheita com imazalil, durante 14 dias de armazenamento a $25^{\circ} \mathrm{C}$.

não apresentados).

Os resultados obtidos na CEAGESP, principalmente com frutos orgânicos, mostram a importância econômica das doenças pós-colheita em citros, uma vez que essas doenças desqualificam a fruta para comercialização. Segundo Paull (22), os danos em pós-colheita não devem ultrapassar os $20 \%$ durante a vida útil dos frutos. A menor ocorrência de doenças nos frutos produzidos em sistema convencional em relação ao orgânico deve-se, provavelmente, ao manejo fitossanitário mais intensivo nos pomares convencionais, com fungicidas de ação mesostêmica/sistêmica. É importante salientar que há poucas opções de insumos certificados na agricultura orgânica para o controle de pragas e doenças, sendo usualmente empregados os agrotóxicos calda sulfocálcica para o manejo das principais pragas e calda bordalesa para o manejo da verrugose (Elsinoëfawcetti e E. australis), da mancha preta (Guignardia citricarpa) e do cancro cítrico (Xanthomonas citri) (21).

A maior incidência de bolor verde em frutos do sistema convencional corrobora os resultados obtidos em frutos cítricos amostrados em packinghouse paulista (9) e também na CEAGESP (10). Os danos decorrentes do bolor verde podem representar mais de $50 \%$ do total de danos em frutos cítricos, durante as fases de transporte, armazenamento e comercialização $(1,5)$. Entretanto, em frutos orgânicos as podridões pedunculares de Lasiodiplodia e phomopsis assumiram maior importância, a exemplo do observado em laranja 'Valência' destinada à exportação (11) e em laranja 'Pêra' comercializada na Central de Abastecimento de Recife (7). A podridão de lasiodiplodia é endêmica em regiões onde as chuvas ocorrem no verão, como Flórida e São Paulo (18). Em Recife, a elevada incidência da doença (11,8\%) foi atribuída à temperatura de armazenamento dos frutos, de $28 \pm 30$ ${ }^{\circ} \mathrm{C}$, considerada ótima para o desenvolvimento de Lasiodiplodia (18); aliada ao uso do etileno para o desverdecimento dos frutos (7), que causa a abscisão precoce do cálice, facilitando a entrada do patógeno que se encontra latente nos tecidos necróticos do botão (2).

A podridão peduncular de Phomopsis normalmente não chega a ser importante em citros, causando perdas de maiores proporções somente em frutos submetidos ao armazenamento refrigerado ou quando demoram a ser comercializados, principalmente a partir da segunda semana de armazenamento (9). Os sintomas iniciais da doença assemelham-se aos da podridão de Lasiodiplodia, porém com o desenvolvimento do patógeno mais lento em direção ao eixo central do fruto.

Os índices de descarte de frutos em pós-colheita são geralmente reflexos dos percentuais de incidência das doenças verificados nessa fase, uma vez que os frutos são desqualificados para comercialização pela simples presença dos sintomas. Assim, a grande diversidade de doenças constatada sugere a necessidade de um manejo fitossanitário adequado no campo e na pós-colheita, visando à obtenção de frutos com melhor qualidade. A redução do inóculo dos principais patógenos pós-colheita pode ser obtida por meio de podas de limpeza e remoção de frutos caídos no pomar. Em pomares convencionais, aplicações de tiofanato metílico em pré-colheita reduziram pela metade a incidência de doenças pós-colheita em cítricos após o armazenamento (24). Para evitar infecções em pós-colheita, principalmente do bolor verde, preconiza-se o manuseio cuidadoso dos frutos durante as operações de colheita, transporte, processamento e armazenamento, uma vez que $P$. digitatum penetra nos fruto por ferimentos (18); a utilização de drencher para a limpeza dos frutos antes de entrarem no packinghouse;

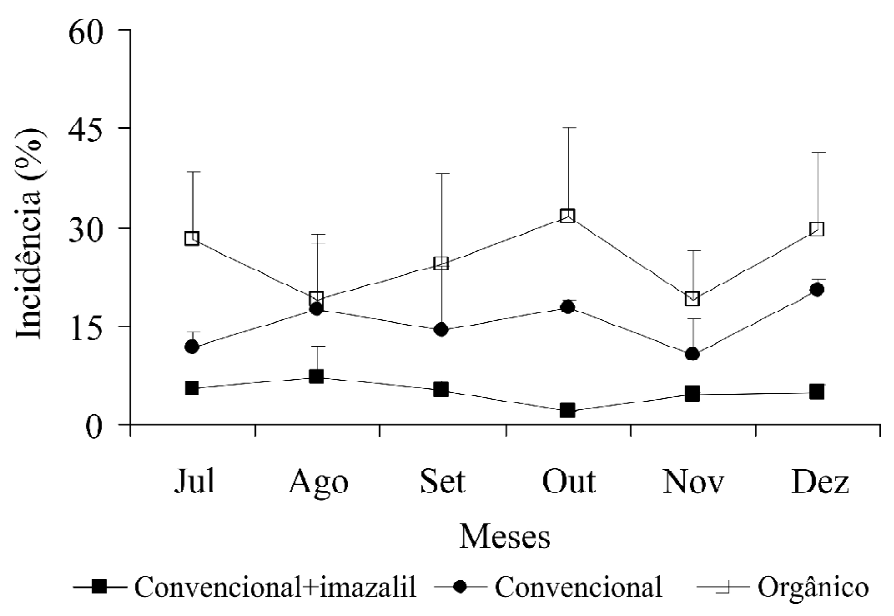

Figura 2. Incidência mensal (\%) de doenças pós-colheita em laranjas 'Pêra' comercializadas na CEAGESP, produzidas em sistema orgânico, convencional e convencional com tratamento pós-colheita com imazalil (média de 2008 e 2009). 
Tabela 3. Incidência de isolados de Penicillium digitatum resistentes in vitro a tiabendazol (TBZ) e imazalil (IZ) oriundos de frutos de laranja 'Pêra' produzida em sistema orgânico, convencional e convencional com tratamento pós-colheita com imazalil ${ }^{1}$.

\begin{tabular}{ccccccc}
\hline \multirow{2}{*}{$\begin{array}{c}P \text {. digitatum } \\
\text { (origem) }\end{array}$} & \multicolumn{2}{c}{ TBZ (10 ppm) } & \multicolumn{2}{c}{ IZ (1 ppm) } & \multicolumn{2}{c}{ TBZ+IZ (10+1 ppm) } \\
\cline { 2 - 7 } & Incid. (\%) & Freq. rel. (\%) & Incid. (\%) & Freq. rel. (\%) & Incid. (\%) & Freq. rel. (\%) \\
\hline Convencional+imazalil & 2,52 & $88,42 \mathrm{a}^{2}$ & 0,31 & $10,88 \mathrm{a}$ & 0,31 & $10,88 \mathrm{a}$ \\
Convencional & 8,70 & $87,34 \mathrm{a}$ & 0,42 & $4,22 \mathrm{~b}$ & 0,16 & $1,61 \mathrm{~b}$ \\
Orgânico & 1,63 & $33,75 \mathrm{~b}$ & 0,17 & $3,52 \mathrm{~b}$ & 0,05 & $1,04 \mathrm{~b}$ \\
\hline
\end{tabular}

${ }^{1}$ Média de 12 amostragens mensais (julho a dezembro de 2008 e 2009) de 200 frutos produzidos em sistema convencional e de 100 frutos orgânicos. ${ }^{2}$ Valores seguidos pela mesma letra minúscula na coluna não diferem entre si, em nível de $5 \%$ pelo teste não paramétrico de comparação de múltiplas proporções.

e a higienização das caixas de colheita e instalações do packinghouse. Do mesmo modo, redução de doenças pós-colheita pode ser obtida pelo emprego de fungicidas em pós-colheita, como observado com imazalil em laranjas 'Pêra' comercializadas na CEAGESP (Tabela 2). Na Flórida, aplicação de fludioxonil em pós-colheita de laranjas reduziu em $76-89 \%$ a incidência de podridão peduncular de lasiodiplodia e em $70-76 \%$ o bolor verde (28). O armazenamento refrigerado dos frutos atrasa consideravelmente o desenvolvimento das doenças, sendo prática importante, especialmente se combinada com o tratamento químico, para a redução das doenças pós-colheita.

\section{Determinação de isolados de Penicillium spp. resistentes a fungicidas}

A frequência relativa de isolados de $P$. digitatum, oriundos de laranjas 'Pêra' com bolor verde, resistentes ao fungicida tiabendazol foi menor quando os frutos foram procedentes de pomares orgânicos em relação aos pomares convencionais (Tabela 3 ). O emprego de imazalil em pós-colheita propiciou uma maior frequência de isolados de $P$. digitatum resistentes a este fungicida e a mistura tiabendazol+imazalil nos frutos. Não foram observadas diferenças quanto à frequência relativa de isolados de $P$. digitatum resistentes a imazalil e a tiabendazol+imazail nos frutos cultivados em sistema orgânico e convencional. De maneira geral foram observadas maiores frequências de isolados de $P$. digitatum resistentes a tiabendazol, comparado a isolados resistentes a imazalil e tiabendazol+imazalil. Estes resultados assemelham-se aos observados em frutos cítricos em Marrocos, onde $37 \%$ dos isolados de $P$. digitatum foram resistentes a tiabendazol, $19 \%$ ao imazalil e $10 \%$ a tiabendazol+imazalil (3).

Estudos indicam que quando populações de patógenos (P. digitatum ou $P$. italicum) são submetidas a exposições repetitivas de fungicidas "sítio-específicos", isolados resistentes podem ser prontamente selecionados $(15,27)$, a exemplo do observado com $P$. digitatum resistente a imazalil e tiabendazol+imazalil em frutos tratados em pós-colheita com imazalil (Tabela 3). Do mesmo modo, a baixa frequência de $P$. digitatum resistente ao imazalil, comparativamente ao tiabendazol, pode estar associada a não utilização do grupo dos imidazóis em pomares citrícolas, além de ser, esse grupo químico considerado de menor risco no desenvolvimento de resistência por parte dos patógenos (8). Imazalil foi considerado efetivo no controle de isolados de $P$. digitatum e $P$. italicum resistentes ao tiabendazol (26, 27). Embora com uma menor incidência absoluta de bolor verde nos frutos tratados com imazalil em pós-colheita, a frequência relativa de isolados de $P$. digitatum resistentes ao tiabendazol não foi inferior em função da aplicação do imazalil em frutos obtidos do sistema de cultivo convencional (Tabelas 2 e 3 ).

A maior ocorrência de $P$. digitatum resistente ao tiabendazol, devese, provavelmente, ao uso no campo dos fungicidas carbendazim e tiofanato metílico, de mesmo modo de ação ao tiabendazol, para o controle da mancha preta dos citros (Guignardia citricarpa), podridão floral (Colletotrichum acutatum), verrugose (Elsinoë spp.) e melanose (Diaphorthe citri). Isolados de P. digitatum resistentes aos benzimidazóis se tornaram comuns em pomares japoneses tratados em pré-colheita por vários anos (17). Embora seja observado que alguns isolados resistentes a tiabendazol são encontrados em pomares e packinghouses onde não se aplicam benzimidazóis $(12,17)$, a exemplo do constatado nos frutos orgânicos, tanto para tiabendazol como imazalil (Tabela 3), o que indica a possibilidade de uma elevada variabilidade genética (25). Segundo Brown (4), em uma população natural de $P$. digitatum, esporos resistentes a tiabendazol são produzidos na razão de 1 a 10 para cada 100 milhões de esporos produzidos, potencialmente, em um único fruto com bolor verde.

Os isolados de $P$. digitatum não apresentaram diferenças de crescimento micelial em meio BDA acrescido ou não de tiabendazol ou imazalil em função da procedência e sistema de cultivo (Tabela 4). Já em meio BDA acrescido de tiabendazol+imazalil os isolados de $P$. digitatum procedentes de frutos orgânicos apresentaram crescimento inferior em relação aos procedentes de frutos convencionais. Não foram verificadas diferenças adaptativas no crescimento micelial dos isolados resistentes a tiabendazol em relação ao crescimento dos isolados na ausência do fungicida, os quais apresentaram uma redução média em

Tabela 4. Crescimento micelial médio $(\mathrm{cm})$ de isolados de Penicillium digitatum, oriundos de frutos de laranja 'Pêra' produzidas em sistema orgânico, convencional e convencional com tratamento pós-colheita com imazalil, em meio batata-dextrose-ágar (BDA) acrescido ou não de tiabendazol (TBZ) e imazalil (IZ) ${ }^{1}$.

\begin{tabular}{ccccc}
\hline \multirow{2}{*}{$\boldsymbol{P}$ digitatum (origem) } & \multicolumn{4}{c}{ Meio de cultivo } \\
\cline { 2 - 5 } & BD A & BDA+TBZ & BDA+IZ & BDA+TBZ + IZ \\
\hline Convencional+imazalil & $7,4 \mathrm{a}^{2}$ & $6,5 \mathrm{a}$ & $3,7 \mathrm{a}$ & $3,7 \mathrm{a}$ \\
Convencional & $8,0 \mathrm{a}$ & $6,2 \mathrm{a}$ & $3,0 \mathrm{a}$ & $3,3 \mathrm{a}$ \\
Orgânico & $8,5 \mathrm{a}$ & $7,2 \mathrm{a}$ & $3,1 \mathrm{a}$ & $1,7 \mathrm{~b}$ \\
\hline CV(\%) & 14,9 & 23,5 & 27,4 & 19,4 \\
\hline
\end{tabular}

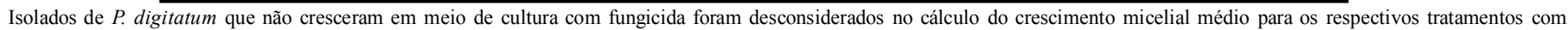
fungicidas. ${ }^{2}$ Valores seguidos pela mesma letra minúscula na coluna não diferem entre si, em nível de $5 \%$ pelo teste de Tukey. 
seu crescimento inferior a $17 \%(12,6-22,5 \%)$, corroborando com os resultados de Holmes \& Eckert (15) e Fischer et al. (12). Os isolados resistentes a imazalil e a tiabendazol+imazail, entretanto, apresentaram redução igual ou superior a $50 \%$ no crescimento micelial na presença dos mesmos. Segundo Holmes \& Eckert (14) e Boubaker et al. (3) a resistência de $P$. digitatum e $P$. italicum ao tiabendazol e imazalil não está associada com a redução na patogenicidade dos isolados.

As condições ambientais favoráveis em packinghouses, a abundante esporulação de Penicillium spp. e o fato de alguns packinghouses empregarem continuamente fungicidas com os mesmos princípios ativos são fatores que contribuem para o desenvolvimento de populações de patógenos resistentes. Em packinghouses californianos de citros a proporção de isolados de $P$. digitatum e $P$. italicum com resistência tripla aos fungicidas imazalil, tiabendazol e ortofenilfenol praticamente duplicou-se em seis anos devido à utilização intensiva destes fungicidas, tanto sequencialmente como em combinação (15). Programas de monitoramento para a sensibilidade a fungicida são frequentemente requeridos visando indicar a necessidade de mudança nos programas de pulverização e, desta forma, prevenir falha de controle. Preconiza-se a combinação de fungicidas com diferentes modos de ação como forma de prevenir o aparecimento de populações resistentes. Segundo Kanetis et al. (16), os produtos azoxistrobina, fludioxonil e pirimetanil podem remediar os problemas de populações de Penicillium spp. resistentes ao tiabendazol e imazalil, entretanto, estes fungicidas não tem registro em pós-colheita para citros no Brasil.

\section{AGRADECIMENTOS}

Os autores agradecem à Fundação de Amparo à Pesquisa do Estado de São Paulo (FAPESP) pelo apoio financeiro concedido ao projeto de pesquisa (Proc. 2007/08519-4), do qual resultou este artigo.

\section{REFERÊNCIAS BIBLIOGRÁFICAS}

1. Agrios, G.N. Plant pathology. 5th ed. Amsterdam. Elsevier Academic Press, 2005.

2. Barmore, C.R.; Brown, G.E. Influence of ethylene on increased susceptibility of oranges to Diplodia natalensis. Plant Disease, Saint Paul, v.69, p. 228-230, 1985 .

3. Boubaker H.; Saadi, B.; Boudyach, E.H.; Benaoumar, A.A. Sensitivity of Penicillium digitatum and P. italicum to imazalil and thiabendazole in Morocco. Plant Pathology Journal, Suwon, v.8, p.152-158, 2009.

4. Brown, G.E. Quality control assessment methodology related to citrus decay control. Lake Alfred: Florida Department of Citrus, Citrus Research and Education Center, 1990. 26 p.

5. Browing, H.; Mcgovern, R.; Jackson, L.; Calvert, D.; Wardowski, W. Florida citrus diagnostic guide. Lake Alfred: Florida Science Source, 1995. 244 p.

6. Camargo, A.M.M.P.; Caser D.V.; Camargo Filho, W.P.; Camargo, F.P.; Coelho, P.J. Área cultivada com agricultura orgânica no estado de São Paulo, 2004. Informações Econômicas, São Paulo, v.36, n.3, p.33-62, 2006.

7. Dantas, S.A.F.; Oliveira, S.M.A.; Michereff, S.J.; Nascimento, L.C.; Gurgel, L.M.S.; Pessoa W.R.L.S. Doenças fúngicas pós-colheita em mamões e laranjas comercializados na Central de Abastecimento do Recife. Fitopatologia Brasileira, Brasília, v.28, p.528-533, 2003.

8. Decker, J. Strategies for avoiding resistance to fungicides. In: Jenkyn, J.E.; Plumb, R.T. (Ed.) Strategies for the control of cereal disease. Oxford: Blackwell, 1985, p.123-133.

9. Fischer, I.H.; Toffano, L.; Lourenço, A.S.; Amorim, L. Caracterização dos danos pós-colheita em citros procedentes de "packi- nhouse". Fitopatologia Brasileira, Lavras, v.32, p.304-310, 2007.

10. Fischer, I.H.; Lourenço, A.S.; Amorim, L. Doenças pós-colheita em citros e caracterização da população fúngica ambiental no mercado atacadista de São Paulo. Tropical Plant Pathology, Lavras, v.33, p.219-226, 2008.

11. Fischer, I.H.; Ferreira, M.D.; Sposito, M.B.; Amorim, L. Citrus postharvest diseases and injuries related to impact on packing lines. Scientia Agricola, Piracicaba, v.66, p.210-217, 2009a.

12. Fischer, I.H.; Afonseca, L.S.; Spósito, M.B.; Amorim, L. Characterisation of the fungal population in citrus packing houses. European Journal of Plant Pathology, Dordrecht, v.123, p.449460, 2009b.

13. França, S.C. Comunidades de fungos micorrízicos arbusculares nos manejos convencional e orgânico de citros e suas interações com Phytophthora parasitica. 2004. 106f. Tese (Doutorado em Agronomia) - Escola Superior de Agricultura "Luiz de Queiroz", Universidade de São Paulo, Piracicaba, 2004.

14. Holmes, G. J.; Eckert, J. W. Relative fitness of imazalil-resistant and -sensitive biotypes of Penicillium digitatum. Plant Disease, Saint Paul, v.79, p.1068-1073, 1995.

15. Holmes, G.J.; Eckert, J.W. Sensitivity of Penicillium digitatum and $P$. italicum to postharvest citrus fungicides in California. Phytopathology, Saint Paul, v.89, p.716-721, 1999.

16. Kanetis, L.; Forster, H.; Adaskaveg, J.E. Optimizing efficacy of new postharvest fungicides and evaluation of sanitizing agents for managing citrus green mold. Plant Disease, Saint Paul, v.92, n.2, p.261-269, 2008.

17. Kuramoto, T. Resistance to benomyl and thiophanate-methyl in strains of Penicillium digitatum and P. italicum in Japan. Plant Disease Reporter, Washington, v.60, p.168-172, 1976.

18. Laranjeira, F.F.; Amorim, L.; Bergamin Filho, A.; Aguilar-Vildoso, C.I.; Della Colleta Filho, H. Fungos, procariotos e doenças abióticas. In: Mattos Junior, D.; De Negri, J.D.; Pio, R.M.; Pompeu Junior, J. (Ed.). Citros. Campinas: Instituto Agronômico, Fundag, 2005. cap. 18, p. 511-558.

19. Macdonald, D.E.; Risse, L.A.; Hillebrand, B.M. Resistance to thiabendazole and benomyl of Penicillium digitatum and P. italicum isolated from citrus fruit from several countries. Journal America Society HortScience, Alexandria, v.194, p.333-335, 1979 .

20. Palou, L.; Usall, J.; Pons, J.; Cerdà, M.C.; Viñas, I. Micoflora em centrales citrícolas de Tarragona. Revista Investigación Agraria: Production Protección Vegetal, Madrid, v.16, p.447-462, 2001.

21. Panzenhagen, N.V.; Koller, O.C.; Vaquil, P.D.; Souza, P.V.D.; Soglio, F.K.D. Aspectos técnico-ambientais da produção orgânica na região citrícola do Vale do Rio Caí, RS. Ciência Rural, Santa Maria, v.38, n.1, p.90-95, 2008.

22. Paull, R.E. Effects of temperature and relative humidity on fresh commodity quality. Postharvest Biology and Technology, Amsterdam, v.15, p.263-277, 1999.

23. Sartori, I.A.; Panzenhagen, N.V.; Soares, D.N.; Soglio, F.K.; Manzete, F. Koller, O.C. Sistemas de cultivo orgânico e convencional em laranjas 'Valência'. Revista Brasileira de Agroecologia, Porto Alegre, v.1, n.1, p.207-211, 2006.

24. Sarvatore, J.J.; Ritenour, M.A. Effectiveness of different fungicides applied preharvest at reducing postharvest decay of fresh Florida citrus. Proceedings of the Florida State Horticultural Society, Florida, v.120, p.281-284, 2007.

25. Schmidt, L.S.; Ghosoph, J.M.; Margosan, D.A.; Smilanick, J.L. Mutation at $\beta$-tubulin codon 200 indicated thiabendazole resistance in Penicillium digitatum collected from California citrus packinghouses. Plant Disease, Saint Paul, v.90, p.765-770, 2006.

26. Smilanik, J.L.; MansouR, M.F.; Margosan, D.A.; Mikota-Gabler, F.; Goodwine, W.R. Influence of $\mathrm{pH}$ and $\mathrm{NaHCO} 3$ on the effectiveness of imazalil to inibit germination or spores of Penicillium digitatum and to control green mold on citrus fruit. Plant Disease, Saint Paul, v.89, p.640-648, 2005.

27. Wild, B.L. Differential sensitivity of citrus green mould isolates 
(Penicillium digitatum Sacc.) to the fungicide isolates imazalil. New Zealand Journal of Crop Horticultural Science, Wellington, v.22, p.167-171, 1994.

28. Zhang, J. The potential of a new fungicide fludioxonil for stem-end rot and green mold control on Florida citrus fruit. Postharvest Biology and Technology, Amsterdam, v.46, p.262-270, 2007.
29. Zar, J.H. Biostatistical analyis. New Jersey: Prentice-Hall, 1999. 663 p.

30. Zhu, J.W.; Xie, Q.Y.; Li, H.Y. Occorrence of imazalil-resistance biotype of Penicillium digitatum in China and the resistance molecular mechanism. Journal of Zhejiang University SCIENCE, Hangzhou, v.7, p.362-365, 2006. 